\title{
Glucocorticoid-induced osteoporosis: Insights for the clinician
}

\section{ABSTRACT}

Glucocorticoids cause significant bone loss, predominantly affecting trabecular bone, with consequent fragility fractures. The risk of fractures is related to the dose and duration of glucocorticoid use, but an increased risk may be observed even at low doses and even in the first month of treatment. Steps to prevent or treat osteoporosis should be considered in all patients who take the equivalent of prednisone at a dose of $2.5 \mathrm{mg}$ or more per day for 3 or more months.

\section{KEY POINTS}

The Fracture Risk Assessment tool (FRAX) includes a yesor-no question about glucocorticoid use, but the formula is based on a medium dose, and the FRAX score should be adjusted upward in patients on high doses and downward in patients on lower doses.

Lifestyle modifications and optimization of calcium and vitamin $D$ intake are recommended for all patients on long-term glucocorticoid therapy.

Bisphosphonates are the first-line drugs for patients at moderate or high fracture risk, based on proven efficacy, safety, and low cost.

Zoledronate (intravenous), teriparatide, and denosumab are second-line options for patients at high risk of fracture on glucocorticoids who cannot tolerate oral bisphosphonates.

Dr. Magrey has disclosed board membership and consulting for Eli Lilly and board membership consulting, teaching, and speaking for Novartis.

doi:10.3949/ccjm.87a.19039
G lucocorticoids are associated with a va$\checkmark$ riety of adverse effects including osteoporosis, with fractures occurring in as many as $50 \%$ of long-term users. ${ }^{1} \mathrm{~A}$ meta-analysis ${ }^{2}$ has shown strong correlations between cumulative dose and loss of bone mineral density, and between daily dose and risk of fracture. The relative risk of fracture is significantly increased even with daily doses as low as $2.5 \mathrm{mg}$ of prednisolone, ${ }^{3}$ and depends on duration of therapy; daily oral prednisone therapy for 3 to 6 months or more has been shown to increase the risk of bone loss in most studies. ${ }^{4,5}$ After glucocorticoids are discontinued, the fracture risk gradually decreases to baseline but may be only partially reversible. ${ }^{4,5}$ The problem affects a great many people, as these drugs are used to treat a variety of inflammatory diseases, and the estimated prevalence of oral use is more than $1 \%$ in the United States and United Kingdom. ${ }^{6-8}$

In 2017, the American College of Rheumatology (ACR) published guidelines for preventing and treating glucocorticoid-induced osteoporosis, with recommendations and algorithms for assessing and categorizing fracture risk, both initially and on follow-up. ${ }^{9}$ This review summarizes the 2017 ACR recommendations, as well as advances in treatment since then.

\section{HOW GLUCOCORTICOIDS DAMAGE BONE}

Bone loss in patients taking glucocorticoids has 2 phases, with rapid loss in the first several months to 1 year followed by a further slower and progressive decline. ${ }^{10}$ The loss is predominantly from trabecular bone, with marked changes in the lumbar spine, but the femoral neck and other sites are also affected. ${ }^{2}$ Even 
at relatively low doses, such as prednisone 2.5 mg daily, glucocorticoids have been shown to cause more than an $8 \%$ decrease in trabecular bone mineral density after just 20 weeks of therapy. ${ }^{11}$

Glucocorticoids damage bone though several mechanisms:

By increasing bone resorption. Initial bone loss is caused by increased bone resorption resulting from upregulation of RANK ligand and suppression of osteoprotegerin. ${ }^{12,13}$ Further, glucocorticoids inhibit gonadotropin secretion, so that serum levels of androgen and estrogen are lower, also causing bone resorption. They also decrease calcium absorption in the intestines, antagonize vitamin $\mathrm{D}$, and decrease renal calcium reabsorption, all resulting in a secondary hyperparathyroid state. ${ }^{14}$

By decreasing bone formation. In cell cultures, glucocorticoids at high doses decrease bone formation by inhibiting osteoblast proliferation, increasing rates of apoptosis of osteoblasts. ${ }^{15}$ They also have been shown to suppress Wnt gene expression in a dose-dependent manner, which in turn suppresses osteogenesis.

By decreasing bone vascularization, likely

Risk begins with daily doses as low

as $\mathbf{2 . 5} \mathbf{~ m g}$ by reducing production of vascular endothelial growth factor by osteoblasts, creating areas of necrosis. ${ }^{16}$ That may explain why reduction in bone strength is greater than that due to reduced bone mass alone. ${ }^{17}$

\section{RISK FACTORS AND FRAX}

Other risk factors can contribute to bone loss in patients taking glucocorticoids. Nonmodifiable risk factors include advanced age, white race, female sex, early menopause, low weight or body mass index, previous fragility fracture, history of rheumatoid arthritis, and a family history of hip fragility fracture. Modifiable risk factors include low calcium or vitamin D intake, estrogen deficiency, immobility, cigarette smoking, and excessive alcohol or caffeine intake. Other comorbid disorders contributing to bone loss must also be taken into account. ${ }^{18}$

\section{The fracture risk calculation}

Released in 2008 by the World Health Organization, the Fracture Risk Assessment Tool (FRAX; https://www.sheffield.ac.uk/FRAX/ index.aspx) has been validated and is com- monly used in clinical practice. It calculates the 10-year probability of a major fracture of the spine, forearm, hip, or shoulder, and the 10 -year probability of a hip fracture. The FRAX models were developed from population-based cohorts from different countries of the world and are further subcategorized by race. It requires the following information:

- Age or date of birth

- Sex

- Weight

- Height

- Bone mineral density of the femoral neck (optional, but recommended for greater accuracy $\left.{ }^{19}\right)$.

Yes-or-no answers are required for:

- Previous fragility fracture

- Hip fracture in a parent

- Smoking status

- Glucocorticoid use (prednisolone $\geq 5 \mathrm{mg} /$ day or the equivalent, for $>3$ months)

- Diagnosis of rheumatoid arthritis

- Alcohol intake of 3 units per day or more

- Disorders associated with secondary osteoporosis-eg, type 1 diabetes, osteogenesis imperfecta in adults, untreated long-standing hyperthyroidism, hypogonadism or early menopause (before age 45), chronic malnutrition or malabsorption, and chronic liver disease.

\section{FRAX risk adjustment for glucocorticoid dose}

The FRAX score for a patient using glucocorticoids is based on a medium dose. Hence, it may underestimate the actual fracture risk in people on higher doses and overestimate the risk in people on lower doses.

Kanis et $\mathrm{a}^{20}$ devised a simple FRAX adjustment (Table 1) based on glucocorticoid dose and age. For example, for those receiving higher doses of glucocorticoids (prednisolone $\geq 7.5 \mathrm{mg}$ /day or equivalent), the adjusted risk of major osteoporotic fracture is $15 \%$ higher in the 60 -to-70 age group and 20\% higher in the 40-to-50 age group compared with unadjusted risk. The FRAX score can also be adjusted without regard for age, as follows:

In patients on medium doses (eg, prednisolone 2.5 to $7.5 \mathrm{mg} /$ day) no adjustment to the FRAX risk is needed.

In patients on low doses (eg, prednisolone $<2.5 \mathrm{mg} /$ day), multiply the unadjusted FRAX 


\section{TABLE 1}

\section{Adjustment in FRAX score by glucocorticoid dose and age}

\begin{tabular}{|c|c|c|c|c|c|c|c|c|}
\hline \multirow[b]{2}{*}{ Dose } & & \multicolumn{7}{|c|}{ Age } \\
\hline & & 40 & 50 & 60 & 70 & 80 & 90 & All \\
\hline & For hip fracture risk & & & & & & & \\
\hline Low $^{\mathrm{a}}$ & Multiply FRAX score by: & 0.60 & 0.60 & 0.50 & 0.40 & 0.70 & 0.70 & 0.65 \\
\hline \multirow[t]{2}{*}{ High $^{b}$} & Multiply FRAX score by: & 1.25 & 1.25 & 1.25 & 1.20 & 1.10 & 1.10 & 1.20 \\
\hline & $\begin{array}{l}\text { For major osteoporoti } \\
\text { fracture risk }\end{array}$ & & & & & & & \\
\hline Low $^{\text {a }}$ & Multiply FRAX score by: & 0.80 & 0.80 & 0.85 & 0.80 & 0.80 & 0.80 & 0.80 \\
\hline High $^{b}$ & Multiply FRAX score by: & 1.20 & 1.20 & 1.15 & 1.15 & 1.10 & 1.10 & 1.15 \\
\hline
\end{tabular}

aPrednisolone $<2.5 \mathrm{mg} /$ day or equivalent.

bPrednisolone $\geq 7.5 \mathrm{mg} /$ day or equivalent.

risk of a major osteoporotic fracture by 0.80 , and multiply the unadjusted risk of a hip fracture by 0.65 .

In patients on high doses (eg, prednisolone $\geq 7.5 \mathrm{mg} /$ day), multiply the unadjusted FRAX risk of major osteoporotic fractures by 1.15 , and multiply the unadjusted FRAX risk of hip fractures by 1.20 .

Example. A 66-year-old woman with rheumatoid arthritis has been taking prednisone $10 \mathrm{mg}$ for 4 months and is expected to continue this dose. According to the unadjusted FRAX score, her 10-year hip fracture risk is $0.9 \%$. This should be multiplied by 1.2 (a 20\% increase), yielding an adjusted FRAX score of $1.08 \%$. The adjustment for glucocorticoid dose suggests that this patient should be treated, as her 10 -year risk of hip fracture is now higher than 1\% (moderate risk).

\section{FRAX caveats}

FRAX cannot be used to estimate the risk of fracture in patients younger than 40. Moreover, FRAX results are partly based on hip bone mineral density (if available), while glucocorticoid use results in more significant loss from the spine (trabecular bone) than from the hip, so the FRAX score may underestimate the true fracture probability. ${ }^{19}$

For patients with discordant bone mineral density in the hip vs the lower spine, the
Foundation for Osteoporosis Research and Education's 10-year risk calculator can be used (https://riskcalculator.fore.org/).

\section{FRACTURE RISK CATEGORIES}

The $\mathrm{ACR}^{9}$ stratifies the risk of fracture in glucocorticoid users into 3 categories:

\section{Low fracture risk}

- Patients age 40 and older with adjusted FRAX risk of less than $10 \%$ for major osteoporotic fracture or less than $1 \%$ for hip fracture

- Patients under age 40 who do not have risk factors other than glucocorticoid exposure.

\section{Moderate fracture risk}

- Patients age 40 and older whose adjusted FRAX risk of major osteoporotic fracture is $10 \%$ to $19 \%$, and whose risk of hip fracture is $1 \%$ to $3 \%$

- Patients under age 40 on glucocorticoids taking $7.5 \mathrm{mg}$ or more daily for at least 6 months, and either hip or spine bone mineral density $\mathrm{Z}$ score below -3 , or rapid bone loss of at least $10 \%$ at the hip or spine over 1 year.

\section{High fracture risk}

- Patients of any age with a history of osteoporotic fracture

- Patients age 40 and older whose T score
Bone is lost rapidly in the first year, followed by a further, slower, progressive decline 


\section{GLUCOCORTICOID-INDUCED OSTEOPOROSIS}

Adults under age $\mathbf{4 0}$

\section{Initial fracture risk assessment}

Clinical fracture risk assessment (within 6 months after starting glucocorticoid treatment)

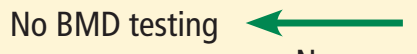

No

History of osteoporotic fracture or other significant risk factors

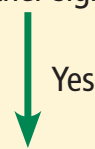

BMD within 6 months of starting glucocorticoid treatment

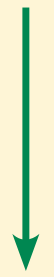

No BMD testing

No

\section{Fracture risk reassessment}

Clinical fracture risk reassessment (every 12 months)
History of osteoporotic fracture or very high glucocorticoid dose or Z score $<-3$ (hip or spine) or $>10 \%$ BMD loss/year (hip or spine or other risk factors?

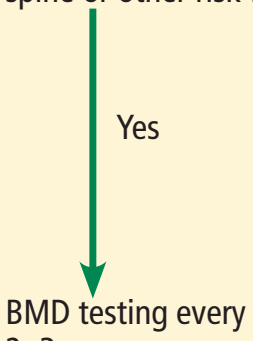

2-3 years
Adults age $\mathbf{4 0}$ and older
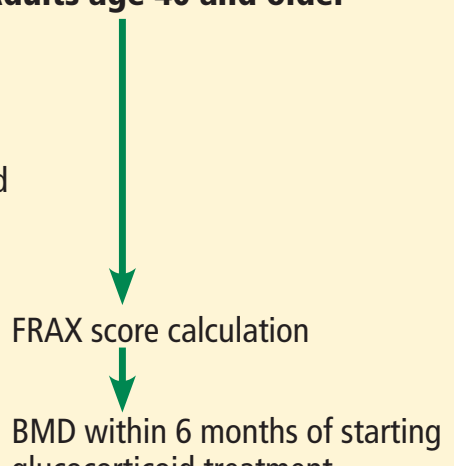
glucocorticoid treatment

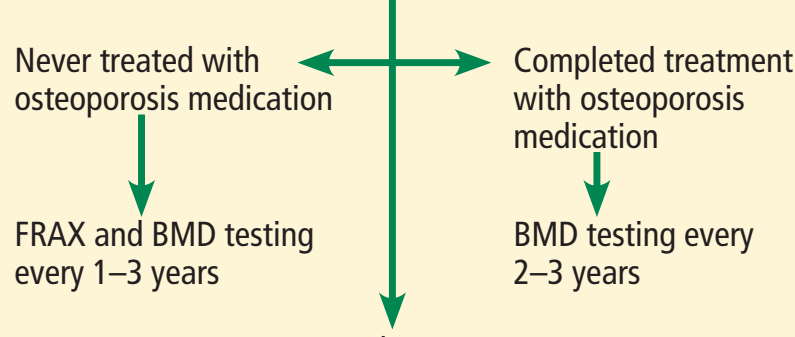

On treatment with osteoporosis medication

History of osteoporotic fracture $\longrightarrow$ No $>18$ months after starting osteoporosis treatment or very high glucocorticoid dose or poor<smiles></smiles>
medication adherence or absorption or other risk factors?

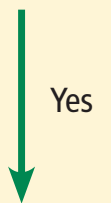

BMD testing every $2-3$ years

$\mathrm{BMD}=$ bone mineral density; FRAX = Fracture Risk Assessment tool (https://www.sheffield.ac.uk/FRAX/tool.aspx?country=9)

Figure 1. An algorithm for initial fracture risk assessment and reassessment in adult patients, based on current guidelines. 
(hip or spine) is -2.5 or lower (in men age 50 and older, and in postmenopausal women)

- Patients with adjusted FRAX risk $20 \%$ or higher for major osteoporotic fracture or $3 \%$ or higher for hip fracture.

Adults age 30 and older on a very high dose of glucocorticoids (eg, prednisone $\geq 30$ mg daily and $>5$-g cumulative dose over the past year) are included in the moderate-tohigh-risk group for treatment.

\section{INITIAL RISK ASSESSMENT}

The initial fracture risk assessment for an adult includes a detailed history reviewing the risk factors for osteoporosis and details of glucocorticoid use including dosages, frequency, and duration. Modifiable, nonmodifiable, and secondary causes of osteoporosis should be reviewed as appropriate, including fall risk.

Physical examination should include weight, height (looking for a decrease), and thorough evaluation of limbs and spine, checking for signs of fracture (bony deformities, spinal tenderness, and kyphosis).

The initial evaluation should be done as soon as possible, but preferably within 6 months after starting glucocorticoid treatment (Figure 1). ${ }^{9}$

For adults younger than 40 , bone mineral density should be measured as soon as possible or within 6 months after starting glucocorticoids if they are at high fracture risk (due to prior osteoporotic fractures) or have other significant osteoporotic risk factors.

For adults 40 and older, the FRAX score should be calculated as soon as possible or within 6 months after starting glucocorticoids and should include the bone mineral density if this testing is available.

\section{REASSESSING FRACTURE RISK}

Every year, a comprehensive evaluation including fracture risk assessment should be completed for patients on glucocorticoids to determine how frequently the bone mineral density needs to be tested (Figure 1$)^{9}$ :

Adults younger than 40 should undergo bone mineral density testing every 2 to 3 years if any of the following features are present:
- High fracture risk (prior osteoporotic fractures)

- High-dose glucocorticoids (eg, prednisone $30 \mathrm{mg}$ or more daily, and cumulative dose 5 g per year)

- Bone mineral density Z score -3 or less (hip or spine)

- Bone mineral density loss from the hip or spine $10 \%$ or more per year, or other significant osteoporotic risk factors.

Adults age 40 and older should be reevaluated based on treatment status. Those on glucocorticoids who have never started osteoporosis treatment (except for vitamin D and calcium) should have their FRAX score calculated, with bone mineral density testing if possible, every 1 to 3 years. Those on glucocorticoids who have completed osteoporosis treatment, or on glucocorticoids and currently on osteoporosis treatment with risk factors for higher fracture risk (including fracture that occurs after 18 months of treatment), should undergo bone mineral density testing every 2 to 3 years.

Additionally, adults age 40 and older should undergo more frequent bone mineral density testing if on high initial doses (eg, prednisone $\geq 30 \mathrm{mg}$ daily and cumulative dose of $5 \mathrm{~g}$ per year) or if at high fracture risk due to prior osteoporotic fracture. Bone mineral density testing can be done closer to every 3 years for patients on lower doses of glucocorticoids and without any other osteoporotic risk factors or who have higher bone mineral densities.

\section{RECOMMENDATIONS FOR TREATMENT}

The $\mathrm{ACR}^{9}$ made its 2017 recommendations after rating the evidence of benefit vs harm for the different treatment options and thoroughly reviewing the literature, after which an expert panel of rheumatologists and internists reached a decision by consensus. Most of the recommendations were conditional, owing to uncertain evidence.

\section{For all:}

\section{Lifestyle, calcium, and vitamin D}

All adults taking the equivalent of prednisone $2.5 \mathrm{mg}$ or more daily for 3 or more months should incorporate lifestyle changes to optimize their bone density, eg, follow a healthy

\section{Evaluate \\ as soon as possible, preferably within 6 months after starting glucocorticoid treatment}


TABLE 2

\section{Treatment based on age and fracture risk}

\begin{tabular}{ll} 
Fracture risk & Treatment \\
\hline Low & Calcium and vitamin D \\
Moderate or high & Calcium and vitamin D \\
& and \\
& $\begin{array}{l}\text { An oral bisphosphonate } \\
\text { or one of the following } \\
\text { (in order of preference) }\end{array}$
\end{tabular}

An intravenous bisphosphonate

Teriparatide

Denosumab

Raloxifene (postmenopausal women)

Based on information in reference 9 . diet, maintain a normal weight (body mass index), stop smoking, limit alcohol intake to less than 3 units/day, engage in low-impact weight-bearing exercises, and take measures to prevent falls.

In addition, these patients should optimize their calcium and vitamin D intake. ${ }^{9}$ Vitamin D helps increase osteoblastogenesis and intestinal absorption of calcium, and glucocorticoids counteract this. ${ }^{21} \mathrm{~A}$ metaanalysis disclosed that vitamin D and calcium supplementation prevented bone loss at the lumbar spine and forearm in glucocorticoid-treated patients; the effect was modest but clinically and statistically significant. ${ }^{22}$ The recommended daily vitamin D intake is about 600 to 800 international units, with a serum level $20 \mathrm{ng} / \mathrm{mL}$ or higher as the goal. Calcium intake, preferably through diet, should be in the range of 1,000 to $1,200 \mathrm{mg}$ daily.

The recommendations for lifestyle, calcium intake, and vitamin D intake are conditional due to indirect and low-quality evidence in glucocorticoid users.

\section{PHARMACOLOGIC TREATMENT}

The guidelines ${ }^{9}$ recommended pharmacologic treatment in addition to the above measures in patients at moderate or high risk of frac- tures, including patients of any age who have had a previous osteoporotic fracture.

An oral bisphosphonate is the first choice, intravenous bisphosphonate the second choice, teriparatide the third choice, and denosumab the fourth (Table 2). This ranking was based on the opinions of the ACR guideline voting panel. The panel recommended oral bisphosphonates as a first choice and parenteral bisphosphonates as a second choice after comparing data about absolute fracture reduction, harms (toxicity and inconvenience of daily injections), and costs (Table 3). A purely evidence-based ranking was not possible since the number of comparative studies was small.

\section{Bisphosphonates}

A Cochrane review of 27 randomized controlled trials ${ }^{23}$ found high-quality evidence that bisphosphonates reduce the risk of vertebral fractures in glucocorticoid-induced osteoporosis, with data extending to 24 months of use, and prevent bone loss at both the lumbar spine and femoral neck. The effect of glucocorticoids on nonvertebral fractures was minimal to none.

Alendronate has been shown to increase bone mineral density in both the lumbar spine and hip in patients taking glucocorticoids and also to reduce the rate of new vertebral fractures. ${ }^{24-27}$

Risedronate similarly was shown in a randomized, placebo-controlled trial to significantly increase bone mineral density. ${ }^{28}$

Intravenous bisphosphonates (eg, zoledronic acid, pamidronate) have also been shown to significantly reduce the rates of nonvertebral fractures, vertebral fractures, and hip fractures.

A meta-analysis of 8 randomized, placebocontrolled trials involving 13,335 patients showed that treatment with zoledronic acid significantly reduced the incidences of nonvertebral fractures, vertebral fractures, and hip fractures. Zoledronic acid was also associated with significant improvement in bone mineral density in the lumbar spine, total hip, femoral neck, and trochanter. ${ }^{29}$ However, the incidence of any adverse event was higher in the zoledronic acid group than in the control group. 
A randomized controlled trial ${ }^{30}$ showed that intravenous zoledronic acid was superior to risedronate in increasing the bone mineral density of the lumbar spine for both treatment and prevention of osteoporosis.

Oral bisphosphonates are cost-effective and considered first-line agents for glucocorticoid-induced osteoporosis. However, intravenous zoledronic acid is superior to oral bisphosphonates and may be preferable in certain patient groups if better compliance is required or fracture risk is high. ${ }^{8}$

\section{Teriparatide}

Teriparatide is a synthetic analogue of parathyroid hormone that activates the Wnt/ beta-catenin pathway in osteoblasts, thereby increasing bone formation. Since inhibition of bone formation is a key mechanism in glucocorticoid-induced osteoporosis, anabolic agents such as teriparatide may be pivotal in its treatment.

Compared with alendronate in treating glucocorticoid-induced osteoporosis in a 36-month randomized controlled trial, ${ }^{31}$ teriparatide was associated with greater increases in bone mineral density in the spine, hip, and femoral neck and with fewer new vertebral fractures. Also, a meta-analysis in 2016 showed teriparatide was efficacious in preventing vertebral fractures in glucocorticoid users. ${ }^{32}$

Despite data that teriparatide reduces the risk of radiographic vertebral fractures more than bisphosphonates do, the 2017 ACR guidelines ${ }^{9}$ recommended it as a second option after bisphosphonates, in view of its higher cost and its inconvenient route of administration (daily injections). ${ }^{33}$ However, we recommend that it be considered as a first-line option in patients who have at least one grade 2 or higher (on a scale of 1 to 4 ) vertebral fracture, based on literature review.

Bone is rapidly lost after teriparatide is discontinued, so an antiresorptive agent should be started soon thereafter if appropriate. ${ }^{25}$

\section{Denosumab}

Denosumab is a fully humanized monoclonal antibody against RANK ligand with a potent antiresorptive effect, resulting in higher bone mineral density at the lumbar spine and total hip and lower risk of new fractures in patients on glucocorticoids. ${ }^{34}$

\section{TABLE 3}

\section{Considerations regarding osteoporosis medications}

\section{Oral bisphosphonates}

Preferred because of safety, low cost, and lack of evidence of superior antifracture benefits from other osteoporosis medications

Avoid in patients with gastroesophageal reflux disease or esophagitis Intravenous bisphosphonates

Higher risk with intravenous infusion (than with oral bisphosphonate therapy) of hypersensitivity reaction, acute-phase reaction (influenzalike illness), hypocalcemia

Longer half-life

Consider for better adherence, given no weekly pill burden

\section{Teriparatide}

Expensive; burden of therapy with daily injections

Limited to 2 years of therapy

Caution in patients with urolithiasis

\section{Denosumab}

Lack of safety data in premenopausal women

Hypersensitivity reaction, infection risk

The safety and efficacy of denosumab in treating glucocorticoid-induced osteoporosis were evaluated in the 12 -month primary analysis of a 2-year, randomized, multicenter, double-blind, parallel-group, active-controlled study in 795 patients. ${ }^{35}$ Patients received either oral risedronate $5 \mathrm{mg}$ daily or denosumab $60 \mathrm{mg}$ subcutaneously every 6 months for 1 year. Denosumab was noninferior (the primary outcome) and superior (a secondary outcome) to risedronate at 1 year in its effect on lumbar bone mineral density in patients who had been on glucocorticoids for at least 3 months or even less than 3 months. Given these findings, denosumab can be used in patients in whom bisphosphonates are contraindicated.

A post hoc analysis revealed that the vertebral fracture rate increased to the level seen in the untreated population after denosumab was discontinued, which needs to be kept in mind when choosing this treatment. ${ }^{36}$ 
The US Food and Drug Administration has approved denosumab for treating glucocorticoid-induced osteoporosis in men and women at high risk of fractures who are either initiating or continuing glucocorticoids in a daily dosage equivalent to $7.5 \mathrm{mg}$ or more of prednisone and are expected to remain on them for at least 6 months. (This approval came after the ACR guidelines were written.)

\section{TREATMENT IN SPECIAL POPULATIONS}

The ACR guidelines included recommendations for initial treatment in special populations. ${ }^{9}$

\section{Women}

In women at moderate to high risk who have childbearing potential, treatment is recommended for those who do not plan on becoming pregnant while receiving osteoporosis medication and are either sexually inactive or using birth control.

- Treat with an oral bisphosphonate rather than calcium and vitamin D alone

- Second-line therapy is teriparatide

- For patients at high risk for whom oral bisphosphonates and teriparatide are not appropriate, consider intravenous bisphosphonates or denosumab (in that order of preference), but consider the potential fetal risks with both options.

These recommendations are conditional, based on low-quality evidence. They have been extrapolated from other treatment groups. In women who are pregnant:

- Optimize calcium, vitamin D, and lifestyle modifications

- No other osteoporosis medications are recommended in this group, given the lack of safety data.

\section{Young patients}

In adults age 30 or older receiving high-dose glucocorticoids (eg, initial dose of prednisone $30 \mathrm{mg}$ per day or higher and cumulative dose higher than $5 \mathrm{~g}$ in 1 year):

- Treat with an oral bisphosphonate rather than calcium and vitamin D alone

- Treat with an oral bisphosphonate rather than an intravenous bisphosphonate, teriparatide, or denosumab

- If bisphosphonates are not appropriate, other treatments are available (Table 2). These recommendations are conditional.

\section{Organ transplant recipients}

For adults with organ transplants who are treated with glucocorticoids, treatment is the same as for everyone else (Table 2) if the glomerular filtration rate is at least $30 \mathrm{~mL} /$ minute and there is no evidence of metabolic bone disease. Renal transplant patients should be evaluated by a metabolic bone disease expert.

The ACR and others, ${ }^{9,37}$ did not recommend denosumab in transplant patients due to lack of safety data when used along with other immunosuppressive medications. However, in a study in 63 organ transplant recipients (15 diabetic patients who received simultaneous kidney and pancreas transplants, 34 patients who received kidney transplants, and 14 patients with liver grafts), denosumab was well tolerated (without serious adverse effects or infections). It improved bone mineral density in the lumbar spine and proximal femur, proving to be a successful option for transplant patients. ${ }^{38}$

\section{FOLLOW-UP TREATMENT RECOMMENDATIONS}

No studies have evaluated the duration of osteoporosis treatment in glucocorticoidinduced osteoporosis. The 2017 ACR guidelines, however, have provided some framework for treatment duration.

In adults age 40 and older taking an oral bisphosphonate, switching the therapy is conditionally recommended if a fracture is sustained after at least 18 months of treatment or if bone mineral density loss is greater than $10 \%$ per year. If the treatment failure is from poor absorption or adherence, then intravenous bisphosphonates should be considered.

For adults age 40 and older who have completed 5 years of oral bisphosphonate treatment and are at moderate or high risk of fracture, treatment can be:

- Continued

- Switched to an intravenous bisphosphonate if there is an absorption or adherence problem

- Switched to another class of medication. Adults age 40 and older on osteoporosis medication, calcium, and vitamin D whose glucocorticoid treatment is stopped and at low risk should discontinue the medication. However, calcium and vitamin D should be contin- 
ued. These recommendations are conditional.

Adults age 40 and older on osteoporosis medication, calcium, and vitamin D whose glucocorticoid treatment has stopped and at moderate or high risk should complete the osteoporosis medication treatment. This recommendation is conditional for moderate-risk patients but strong for high-risk patients.

\section{SUMMING UP}

Glucocorticoid-induced osteoporosis is a major cause of bone loss and consequent fragil-

\section{REFERENCES}

1. Weinstein RS. Glucocorticoid-induced osteoporosis and osteonecrosis. Endocrinol Metab Clin North Am 2012; 41(3):595-611. doi:10.1016/j.ecl.2012.04.004

2. van Staa TP, Leufkens HG, Cooper C. The epidemiology of corticosteroid-induced osteoporosis: a meta-analysis. Osteoporos Int 2002; 13(10):777-787. doi:10.1007/s001980200108

3. Van Staa TP, Leufkens HG, Abenhaim L, Zhang B, Cooper C. Use of oral corticosteroids and risk of fractures. J Bone Miner Res 2000; 15(6):993-1000. doi:10.1359/jbmr.2000.15.6.993

4. De Vries F, Bracke M, Leufkens HG, Lammers JW, Cooper C, Van Staa TP. Fracture risk with intermittent highdose oral glucocorticoid therapy. Arthritis Rheum 2007; 56(1):208-214. doi:10.1002/art.22294

5. Raterman HG, Bultink IEM, Lems WF. Current treatments and new developments in the management of glucocorticoid-induced osteoporosis. Drugs 2019; 79(10):1065-1087. doi:10.1007/s40265-019-01145-6

6. Overman RA, Yeh JY, Deal CL. Prevalence of oral glucocorticoid usage in the United States: a general population perspective. Arthritis Care Res (Hoboken) 2013 65(2):294-298. doi:10.1002/acr.21796

7. Overman RA, Toliver JC, Yeh JY, Gourlay ML, Deal CL. United States adults meeting 2010 American College of Rheumatology criteria for treatment and prevention of glucocorticoid-induced osteoporosis. Arthritis Care Res (Hoboken) 2014; 66(11):1644-1652. doi:10.1002/acr.22346

8. Walsh LJ, Wong CA, Pringle M, Tattersfield AE. Use of oral corticosteroids in the community and the prevention of secondary osteoporosis: a cross sectional study. BMJ 1996; 313(7053):344-346. doi:10.1136/bmj.313.7053.344

9. Buckley L, Guyatt G, Fink HA, et al. 2017 American College of Rheumatology guideline for the prevention and treatment of glucocorticoid-induced osteoporosis. Arthritis Rheumatol 2017; 69(8):1521-1537. doi:10.1002/art.40137

10. LoCascio V, Bonucci E, Imbimbo B, et al. Bone loss in response to long-term glucocorticoid therapy. Bone Miner 1990; 8(1):39-51. doi:10.1016/0169-6009(91)90139-q

11. Laan RF, van Riel PL, van de Putte LB, van Erning $L J$, van't Hof MA, Lemmens JA. Low-dose prednisone induces rapid reversible axial bone loss in patients with rheumatoid arthritis. A randomized, controlled study. Ann Intern Med 1993; 119(10):963-968. doi:10.7326/0003-4819-119-10-199311150-00001

12. Hofbauer LC, Gori F, Riggs BL, et al. Stimulation of osteo- ity fractures. Despite high risk of fractures and comprehensive recommendations for treatment, most patients on glucocorticoids are not treated for it.

In addition to lifestyle modifications, pharmacologic treatment is recommended in both men and women taking glucocorticoids based on dose and duration of use. The first-line agents continue to be oral bisphosphonates. Intravenous bisphosphonates, teriparatide, and denosumab are recommended for patients with high risk for fractures or unable to tolerate oral bisphosphonates.

protegerin ligand and inhibition of osteoprotegerin production by glucocorticoids in human osteoblastic lineage cells: potential paracrine mechanisms of glucocorticoidinduced osteoporosis. Endocrinology 1999; 140(10):43824389. doi:10.1210/endo.140.10.7034

13. Kondo T, Kitazawa R, Yamaguchi A, Kitazawa S. Dexamethasone promotes osteoclastogenesis by inhibiting osteoprotegerin through multiple levels. J Cell Biochem 2008; 103(1):335-345. doi:10.1002/jcb.21414

14. Patschan D, Loddenkemper K, Buttgereit F. Molecular mechanisms of glucocorticoid-induced osteoporosis. Bone 2001; 29(6):498-505. doi:10.1016/s8756-3282(01)00610-x

15. Uda Y, Azab E, Sun N, Shi C, Pajevic PD. Osteocyte mechanobiology. Curr Osteoporos Rep 2017; 15(4):318-325. doi:10.1007/s11914-017-0373-0

16. Lane NE. Glucocorticoid-induced osteoporosis: new insights into the pathophysiology and treatments. Curr Osteoporos Rep 2019; 17(1):1-7. doi:10.1007/s11914-019-00498-x

17. Weinstein RS. Glucocorticoids, osteocytes, and skeletal fragility: the role of bone vascularity. Bone 2010; 46(3):564-570. doi:10.1016/j.bone.2009.06.030

18. Fitzpatrick LA. Secondary causes of osteoporosis. Mayo Clin Proc 2002; 77(5):453-468. doi:10.4065/77.5.453

19. Buckley L, Humphrey MB. Glucocorticoid-induced osteoporosis. N Engl J Med 2018; 379(26):2547-2556. doi:10.1056/NEJMcp1800214

20. Kanis JA, Johansson H, Oden A, McCloskey EV. Guidance for the adjustment of FRAX according to the dose of glucocorticoids. Osteoporos Int 2011; 22(3):809-816. doi:10.1007/s00198-010-1524-7

21. Gröber U, Kisters K. Influence of drugs on vitamin $D$ and calcium metabolism. Dermatoendocrinol 2012; 4(2):158-166. doi:10.4161/derm.20731

22. Homik J, Suarez-Almazor ME, Shea B, Cranney A, Wells G, Tugwell P. Calcium and vitamin D for corticosteroidinduced osteoporosis. Cochrane Database Syst Rev 2000; (2):CD000952. doi:10.1002/14651858.CD000952

23. Allen CS, Yeung JH, Vandermeer B, Homik J. Bisphosphonates for steroid-induced osteoporosis. Cochrane Database Syst Rev 2016; 10:CD001347. doi:10.1002/14651858.CD001347.pub2

24. Axelsson KF, Nilsson AG, Wedel H, Lundh D, Lorentzon M. Association between alendronate use and hip fracture risk in older patients using oral prednisolone. JAMA 2017; 318(2):146-155. doi:10.1001/jama.2017.8040

25. Emkey R, Delmas PD, Goemaere S, et al. Changes in bone mineral density following discontinuation or continuation of alendronate therapy in glucocorticoidtreated patients: a retrospective, observational study. 
Arthritis Rheum 2003; 48(4):1102-1108. doi:10.1002/art.10861

26. Saag KG, Emkey R, Schnitzer TJ, et al. Alendronate for the prevention and treatment of glucocorticoid-induced osteoporosis. Glucocorticoid-Induced Osteoporosis Intervention Study Group. N Engl J Med 1998; 339(5):292-299. doi:10.1056/NEJM199807303390502

27. Adachi JD, Saag KG, Delmas PD, et al. Two-year effects of alendronate on bone mineral density and vertebral fracture in patients receiving glucocorticoids: a randomized, double-blind, placebo-controlled extension trial. Arthritis Rheum 2001; 44(1):202-211. doi:10.1002/15290131(200101)44:1<202::AID-ANR27>3.0.CO;2-W

28. Cohen S, Levy RM, Keller M, et al. Risedronate therapy prevents corticosteroid-induced bone loss: a twelvemonth, multicenter, randomized, double-blind, placebo-controlled, parallel-group study. Arthritis Rheum 1999; 42(11):2309-2318. doi:10.1002/15290131(199911)42:11<2309::AID-ANR8>3.0.CO;2-K

29. Wang C. Efficacy and safety of zoledronic acid for treatment of postmenopausal osteoporosis: a metaanalysis of randomized controlled trials. Am J Ther 2017; 24(5):e544-e552. doi:10.1097/MJT.0000000000000415

30. Reid DM, Devogelaer JP, Saag K, et al; HORIZON investigators. Zoledronic acid and risedronate in the prevention and treatment of glucocorticoid-induced osteoporosis (HORIZON): a multicentre, double-blind double-dummy, randomised controlled trial. Lancet 2009; 373(9671):1253-1263. doi:10.1016/S0140-6736(09)60250-6

31. Saag KG, Zanchetta JR, Devogelaer JP, et al. Effects of teriparatide versus alendronate for treating glucocorticoid-induced osteoporosis: thirty-six-month results of a randomized, double-blind, controlled trial. Arthritis Rheum 2009; 60(11):3346-3355. doi:10.1002/art.24879

32. Amiche MA, Albaum JM, Tadrous M, et al.,Efficacy of osteoporosis pharmacotherapies in preventing fracture among oral glucocorticoid users: a network meta-analysis. Osteoporos Int 2016; 27(6):1989-1998. doi:10.1007/s00198-015-3476-4

33. Buckley L, Guyatt G, Fink HA, McAlindon T. Reply. Arthritis Care Res (Hoboken) 2018; 70(6):950-951. doi:10.1002/acr.23416

34. Dore RK, Cohen SB, Lane NE, et al; Denosumab RA Study Group. Effects of denosumab on bone mineral density and bone turnover in patients with rheumatoid arthritis receiving concurrent glucocorticoids or bisphosphonates. Ann Rheum Dis 2010; 69(5):872-875. doi:10.1136/ard.2009.112920

35. Saag KG, Wagman RB, Geusens $\mathbf{P}$, et al. Denosumab versus risedronate in glucocorticoid-induced osteoporosis: a multicentre, randomised, double-blind, active-controlled, double-dummy, non-inferiority study. Lancet Diabetes Endocrinol 2018; 6(6):445-454. doi:10.1016/S2213-8587(18)30075-5

36. Cummings SR, Ferrari S, Eastell R, et al. Vertebral fractures after discontinuation of denosumab: a post hoc analysis of the randomized placebo-controlled FREEDOM trial and its extension. J Bone Miner Res 2018; 33(2):190198. doi:10.1002/jbmr.3337

37. Hsu E, Nanes M. Advances in treatment of glucocorticoid-induced osteoporosis. Curr Opin Endocrinol Diabetes Obes 2017: 24(6):411-417. doi:10.1097/MED.0000000000000368

38. Brunova J, Kratochvilova S, Stepankova J. Osteoporosis therapy with denosumab in organ transplant recipients. Front Endocrinol (Lausanne) 2018; 9:162. doi:10.3389/fendo.2018.00162

Address: Marina N. Magrey, MD, Department of Rheumatology, Metrohealth Medical Center, 2500 MetroHealth Drive, Cleveland, $\mathrm{OH} 44109 ;$ mmagrey@metrohealth.org 